\title{
Molecular spintronics
}

Cite as: J. Appl. Phys. 125, 240401 (2019); doi: 10.1063/1.5113900

Submitted: 9 June 2019 . Accepted: 9 June 2019 .

Published Online: 26 June 2019

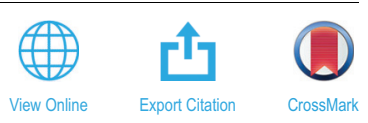

Marco Gobbi, ${ }^{7}$ (D) Miguel A. Novak, ${ }^{2}$ and Enrique Del Barco ${ }^{3}$ (D)

\author{
AFFILIATIONS \\ ${ }^{1}$ Centro de Fisica de Materiales (CSIC-UPV/EHU), Paseo Manuel de Lardizabal 5, E-20018 Donostia-San Sebastián, Spain \\ ${ }^{2}$ Instituto de Física, Universidade Federal do Rio de Janeiro, Rio de Janeiro RJ-21941-972, Brazil \\ ${ }^{3}$ Department of Physics, University of Central Florida, Orlando, Florida 32816, USA
}

\begin{abstract}
Molecules emerged as intriguing materials for spintronics due to their weak spin scattering mechanisms and long spin lifetime, which permit them to retain the spin information over relatively long times. Due to this peculiarity, thin molecular films were initially used as passive spin-transporting media in hybrid spin-valve architectures. Over time, molecules acquired a more active role for spintronics, enabling the generation of hybrid electronic states with peculiar magnetic textures. Moreover, molecular magnets offer the opportunity to manipulate individual spins, representing a two-level system ideal for quantum computing. Evolving at the crossroad between these topics, molecular spintronics has become a broad and interdisciplinary research area in which the paths of conventional spintronics, organic electronics, surface science, and single-molecule devices are converging, offering innovative solutions for next-generation spintronics and quantum technology. For these reasons, the Journal of Applied Physics has assembled a special topic covering different approaches on this exciting subject.
\end{abstract}

Published under license by AIP Publishing. https://doi.org/10.1063/1.5113900

\section{INTRODUCTION}

Molecular spintronics targets the control over the spin degree of freedom in molecular materials for the demonstration of new devices that rely on specific molecular functionalities. It represents an exciting field in which the interests and efforts of scientists active in different research areas converge, motivating the publication of a collection of works assembled in a special topic in the Journal of Applied Physics. To fully appreciate the advances described in the featured works, we provide a brief description of the state of art, highlighting some important discoveries that have marked the evolution of the field in the recent past.

The initial interest in molecules for spintronics was motivated by their weak spin scattering mechanisms and long spin lifetime, which made them the ideal materials to store and manipulate the spin information-essential in spintronics-over relatively long time scales. ${ }^{1}$ Hence, thin films of organic molecules were initially employed as a spin-transporting medium separating ferromagnetic contacts in hybrid organic/inorganic spin valves. ${ }^{2}$ Through this device approach, efficient spin injection and transport into organic films were demonstrated, ${ }^{2}$ generating excitement in the spintronic community and marking the birth of organic spintronics. In this effort, different spintronic devices based on molecular thin films were demonstrated with performances comparable to their fully inorganic counterpart, $^{3,4}$ and further specific molecular capabilities were shown to provide additional functionalities to spintronic devices, enabling, for instance, the demonstration of spin-photovoltaic devices that can be operated with minimal energy consumption, ${ }^{5}$ offering a brilliant solution for low-power spin-electronics.

The understanding that spin-related effects taking place at the organic/inorganic interface determine the performances of hybrid spin valves marked another milestone in the field. ${ }^{6,7}$ In particular, the magnetic properties of ferromagnetic surfaces are modified by the presence of molecules, as new hybrid states with tailored spin textures are generated-the so-called spinterfaces. ${ }^{7}$ In this way, surface science studies at the molecular level complement the investigation of macroscopic devices ${ }^{8}$ and provide valuable insight into the details of spin injection. Taking a step further, molecules can be chosen to design novel magnetic surfaces with the desired spin properties. ${ }^{9,10}$ In this process, the role of molecules has evolved from a passive spin-transporting medium to an active tool capable of tailoring surface magnetism and generating peculiar magnetic states. ${ }^{8}$ In turn, since the details of the interface between magnetic and nonmagnetic materials determine the output signals of spin valves, a precise control over surface magnetism offers the possibility to improve their performances and eventually achieve better-performing hybrid magnetic field sensors.

The ultimate step toward the miniaturization of molecular spintronics foresees the control over the spin degree of freedom in 
individual molecules. ${ }^{11}$ At this level, quantum phenomena dominate the charge and spin transport, making single-molecule spintronics a promising route for the development of novel quantum technologies. Within this approach, a particular interest is dedicated to the spin and charge quantum transport across single-molecule magnets and other magnetic molecules ${ }^{12}$ that are characterized by slow spin dynamics at low temperature ${ }^{13}$ and represent a promising approach to quantum bits. Indeed, the Grover quantum search algorithm has recently been demonstrated using the nuclear spin state of a molecular magnet placed between the leads of a singleelectron transistor. ${ }^{14}$

Finally, the development of molecular spintronics has triggered theoretical investigation at very different levels, ${ }^{15}$ from modeling of the spin transport in macroscopic molecular films to the first-principles simulation of molecules on surfaces and quantum effects in single-molecule junctions.

The achievements mentioned in this introduction provide a succinct overview of the most active research lines in the field of molecular spintronics. Building on this solid foundation, the collection of works published in the special topic in the Journal of Applied Physics described below includes several significant advances toward the design of tailored materials and interfaces, as well as their integration in innovative devices for spintronics and quantum computing.

\section{SUMMARY OF AREAS COVERED}

The Molecular Spintronics special topic in the Journal of Applied Physics offers an overview of the most active research areas currently under investigation in the broad field of molecular spintronics. In particular, featured topics include spin readout and manipulation in single-molecule magnets, ${ }^{16-18}$ hybrid spin states and magnetic coupling at molecule/metal interfaces, ${ }^{19-22}$ spin transport across ultrathin molecular monolayers ${ }^{23}$ and molecular films with novel experimental techniques, ${ }^{24}$ and novel promising materials for molecular spintronics such as organic radicals. ${ }^{25,26}$

A particular emphasis is devoted to single-molecule spintronics for quantum computing, ${ }^{16-18}$ with two works demonstrating the potential of one among the prototypical single-molecule magnets $\left(\mathrm{TbPc}_{2}\right)$ as quantum bits. Molecular magnets are quantum entities characterized by two stable spin states, separated by a potential barrier. Therefore, reversing the spin requires a certain amount of energy. In a tutorial article, Balestro and co-workers ${ }^{16}$ explain how to monitor the reversal of the electron spin in a $\mathrm{TbPc}_{2}$ single-molecule spin transistor. Additionally, they show that the application of microwave pulses causes the spin to flip between two states, providing a way to switch the spin controllably. ${ }^{16}$ In a related work, Wernsdorfer and co-workers investigate the origin of tunneling dynamics in molecular magnets. ${ }^{18}$ In these compounds, tunneling is particularly important, since it provides an underbarrier path to overcome the potential barrier between spin states. Focusing on $\mathrm{TbPc}_{2}$ as a case example, the authors show how nuclear quadrupolar interactions permit to account for the experimentally observed magnetization dynamics at low temperatures. ${ }^{18}$ Such in-depth understanding of the effects determining quantum tunneling represent a key element to guide organic chemists in the design and synthesis of application-oriented molecular magnets.
Fine control over surface magnetism can be achieved at metal/molecule interfaces. Zeppenfeld and co-workers show how the magnetism of a ferromagnetic film is modified by the presence of an adsorbed molecular overlayer. ${ }^{19}$ In their study, they show that the magnetization of an atomically thin Ni film, which points out-of-plane in the case of the pristine film, is switched to in-plane when it is covered by a few monolayers of a paramagnetic molecule. The interface between molecules and an ultrathin molecular layer is the topic of the work by Brambilla and co-workers, ${ }^{21}$ who focus on the interface between chromium oxide monolayers and $\mathrm{C}_{60}$. In this case, a hybrid state appears with peculiar spin polarization. ${ }^{21}$ These works provide nice examples of how molecular decoration can be employed to modify the intrinsic properties of surfaces and thin films, thereby offering the possibility to generate novel hybrid materials with tailored properties, which might be integrated in spintronic devices.

This Molecular Spintronics special topic also covers the spin transport in molecular layers and its application in multifunctional devices. Vardeny et al. study how the curvature of different fullerenes $\left(\mathrm{C}_{60}, \mathrm{C}_{70}, \mathrm{C}_{84}\right)$ affects the spin transport to validate a prediction according to which the curvature in carbonbased materials increases the spin-orbit coupling. ${ }^{24}$ With this goal, they employ a spin pumping technique to inject a pure spin current into films of $\mathrm{C}_{60}, \mathrm{C}_{70}$, and $\mathrm{C}_{84}$ and measure the spin diffusion length in films in the different cases. The longest spin diffusion length is achieved in $\mathrm{C}_{84}$, for which the curvature is the lowest; this finding is in agreement with the prediction of curvature-increased spin-orbit coupling in carbon-based materials. ${ }^{24}$ In another work, Lin and co-workers investigate the spin transport across a self-assembled monolayer. ${ }^{23}$ In this study, the authors sandwich an ultrathin molecular film between two ferromagnetic electrodes in a spin-valve architecture. Interestingly, the electrical resistance of such a device can be modified not only by the application of a magnetic field (in analogy to all-inorganic spin valves) but also by the application of a relatively high current through the barrier ${ }^{23}$ (in analogy to the so-called memristors). This result highlights the potential of integrating self-assembled monolayers into future spintronic architectures to buildup multifunctional devices.

Finally, works on the design and characterization of new materials for molecular spintronics are presented. As an example, stable organic radicals are particularly attractive for spintronics, since they possess a nonzero net spin which is magnetically active. Veciana and co-workers study the assembly and electrical characteristics of an electron donor-acceptor radical dyad on graphite. ${ }^{25}$ Remarkably, they find that the presence of a radical in the molecules enhances the electrical conduction by almost two orders of magnitude, making this material highly attractive for spin-electronics. This study might impulse further works to design a suitable device architecture to take full advantage from the unpaired spin in organic radicals.

The variety of the featured topics highlights the multidisciplinarity of molecular spintronics, which is fueled by the joint effort of scientists active in very different research fields, including device physics, quantum computing, surface science, and synthetic or physical chemistry. 


\section{CONCLUSIONS}

Molecular spintronics aims to exploit the unique chemical flexibility offered by molecular design to achieve precise control over the spin degree of freedom in novel device architectures. The Molecular Spintronics special topic in the Journal of Applied Physics focuses on different approaches to this ultimate goal, spanning from materials design to device engineering. Thanks to the high quality of the works and to the contribution of leading scientists in the field, the collection of papers on Molecular Spintronics provides an authoritative assessment of the state of the art of molecular spintronics.

While providing a glimpse of the countless possibilities of molecular spintronics, the collection of works also evidences that many routes remain unexplored. For instance, the novel molecular materials and tailored hybrid interfaces described in Refs. 19-22, 25, and 26 might enable the demonstration of unconventional devices, adding novel functionalities to conventional spintronics and providing a material platform for quantum computing. In this regard, we hope that the featured works will inspire new ideas and boost further investigations and that scientists active in different fields will enjoy the reading.

\section{ACKNOWLEDGMENTS}

M.G. acknowledges support from the European Commission through the Marie Sklodowska-Curie IEF project SUPER2D (No. GA-748971). E.d.B. acknowledges support from the Engineering Division of the American Science Foundation (No. ECCS-1916874). M.A.N. acknowledges the support from the Brazilian agencies CAPES, CNPq, and FAPERJ. The guest editors sincerely thank the staff and editors of the Journal of Applied Physics for putting this special topic together and all the authors for their contributions.

\section{REFERENCES}

${ }^{1}$ V. A. Dediu, L. E. Hueso, I. Bergenti, and C. Taliani, Nat. Mater. 8, 707 (2009).

2Z. H. Xiong, D. Wu, Z. Valy Vardeny, and J. Shi, Nature 427, 821 (2004).

${ }^{3}$ T. S. Santos, J. S. Lee, P. Migdal, I. C. Lekshmi, B. Satpati, and J. S. Moodera, Phys. Rev. Lett. 98, 16601 (2007)

${ }^{4}$ M. Gobbi, A. Bedoya-Pinto, F. Golmar, R. Llopis, F. Casanova, and L. E. Hueso, Appl. Phys. Lett. 101, 102404 (2012).

${ }^{5}$ X. Sun, S. Vélez, A. Atxabal, A. Bedoya-Pinto, S. Parui, X. Zhu, R. Llopis, F. Casanova, and L. E. Hueso, Science 357, 677 (2017).
${ }^{6}$ C. Barraud, P. Seneor, R. Mattana, S. Fusil, K. Bouzehouane, C. Deranlot, P. Graziosi, L. Hueso, I. Bergenti, V. Dediu, F. Petroff, and A. Fert, Nat. Phys. 6, 615 (2010).

${ }^{7}$ S. Sanvito, Nat. Phys. 6, 562 (2010).

${ }^{8}$ M. Cinchetti, V. A. Dediu, and L. E. Hueso, Nat. Mater. 16, 507 (2017).

${ }^{\mathbf{9}}$ K. V. Raman, A. M. Kamerbeek, A. Mukherjee, N. Atodiresei, T. K. Sen, P. Lazić, V. Caciuc, R. Michel, D. Stalke, S. K. Mandal, S. Blügel, M. Münzenberg, and J. S. Moodera, Nature 493, 509 (2013).

${ }^{10}$ F. Al Ma'Mari, T. Moorsom, G. Teobaldi, W. Deacon, T. Prokscha, H. Luetkens, S. Lee, G. E. Sterbinsky, D. A. Arena, D. A. MacLaren, M. Flokstra, M. Ali, M. C. Wheeler, G. Burnell, B. J. Hickey, and O. Cespedes, Nature 524, 69 (2015).

${ }^{11}$ M. Ormaza, P. Abufager, B. Verlhac, N. Bachellier, M.-L. Bocquet, N. Lorente, and L. Limot, Nat. Commun. 8, 1974 (2017).

${ }^{12}$ R. Vincent, S. Klyatskaya, M. Ruben, W. Wernsdorfer, and F. Balestro, Nature 488, 357 (2012).

${ }^{13}$ M. Mannini, F. Pineider, C. Danieli, F. Totti, L. Sorace, P. Sainctavit, M.-A. Arrio, E. Otero, L. Joly, J. C. Cezar, A. Cornia, and R. Sessoli, Nature 468, 417 (2010).

${ }^{14}$ C. Godfrin, A. Ferhat, R. Ballou, S. Klyatskaya, M. Ruben, W. Wernsdorfer, and F. Balestro, Phys. Rev. Lett. 119, 187702 (2017).

${ }^{15}$ A. R. Rocha, V. M. García-suárez, S. W. Bailey, C. J. Lambert, J. Ferrer, and S. Sanvito, Nat. Mater. 4, 335 (2005).

${ }^{16}$ C. Godfrin, S. Lumetti, H. Biard, E. Bonet, S. Klyatskaya, M. Ruben, A. Candini, M. Affronte, W. Wernsdorfer, and F. Balestro, J. Appl. Phys. 125, 142801 (2019).

${ }^{17}$ A. Palii and B. Tsukerblat, J. Appl. Phys. 125, 142901 (2019).

${ }^{18}$ G. Taran, E. Bonet, and W. Wernsdorfer, J. Appl. Phys. 125, 142903 (2019).

${ }^{19}$ M. Denk, D. Queteschiner, M. Hohage, A. Navarro-Quezada, and P. Zeppenfeld, J. Appl. Phys. 125, 142902 (2019).

${ }^{20}$ V. Corradini, A. Candini, D. Klar, R. Biagi, V. De Renzi, A. Lodi Rizzini, N. Cavani, U. del Pennino, H. Wende, E. Otero, and M. Affronte, J. Appl. Phys. 125, 142904 (2019).

${ }^{21}$ A. Brambilla, A. Picone, S. Achilli, G. Fratesi, A. Lodesani, A. Calloni, G. Bussetti, M. Zani, M. Finazzi, L. Duò, and F. Ciccacci, J. Appl. Phys. 125, 142907 (2019).

${ }^{22}$ M. P. Bahlke, P. Wahl, L. Diekhöner, and C. Herrmann, J. Appl. Phys. 125, 142910 (2019).

${ }^{23}$ J.-Y. Hong, S.-H. Chang, K.-H. Ou Yang, P.-C. Yeh, H.-W. Shiu, C.-H. Chen, W.-C. Chiang, and M.-T. Lin, J. Appl. Phys. 125, 142905 (2019).

${ }^{24}$ H. Liu, J. Wang, A. Chanana, and Z. V. Vardeny, J. Appl. Phys. 125, 142908 (2019).

${ }^{25}$ J. Guasch, N. Crivillers, M. Souto, I. Ratera, C. Rovira, P. Samorì, and J. Veciana, J. Appl. Phys. 125, 142909 (2019).

${ }^{26}$ J. Liu, J. Kang, and Y. Wang, J. Appl. Phys. 125, 142906 (2019). 